\title{
Throughput Analysis of Ieee802.11b Wireless Lan With One Access Point Using Opnet Simulator
}

\author{
Isizoh A. N. ${ }^{1}$ \\ Department of Electronic and Computer Engineering, \\ Nnamdi Azikiwe University, Awka, Nigeria.
}

Okide S. O. ${ }^{3}$

Department of Computer Science, Nnamdi Azikiwe University, Awka, Nigeria.

\author{
Nwokoye A. O.C. ${ }^{2}$ \\ Department of Physics and Industrial Physics, \\ Nnamdi Azikiwe University, Awka, Nigeria.
}

\section{Ogu C. D. ${ }^{4}$}

Department of Electronic and Computer Engineering, Nnamdi Azikiwe University, Awka, Nigeria.

\begin{abstract}
This paper analyzes the throughput performance of IEEE 802.11b Wireless Local Area Network (WLAN) with one access point. The IEEE 802.11b is a wireless protocol standard. In this paper, a wireless network was established which has one access point. OPNET IT Guru Simulator (Academic edition) was used to simulate the entire network. Thus the effects of varying some network parameters such as the data-rate, buffer-sizes, and fragmentation threshold were observed on the throughput performance metric. Several simulation graphs were obtained and used to analyze the network performance.
\end{abstract}

Keywords- Data-rate; buffer size; fragmentation threshold; throughput; Media Access Control (MAC).

\section{INTRODUCTION}

A network is a group of devices, such as computers that are connected to each other for the purpose of sharing information and resources. Shared resources can include printers, documents and internet access connections. A network can be wired or wireless. $802.11 \mathrm{~b}$ is one of the IEEE protocol standards for wireless networks. It uses a modulation technique known as Direct Sequence Spread Spectrum (DSSS) [1].

Wireless network has some attributes or parameters such as data-rates, buffer sizes, fragmentation threshold (FTS), etc. It also has some qualities of service or metrics like the Throughput, Delay, Media access delay, Data dropped, Retransmission attempts, etc. But analysis here is only for throughput.

\section{A. Throughput Analysis}

In a wireless network, system throughput is defined as the fraction of time that a channel is used to successfully transmit payload bits.

Throughput can be obtained by analyzing the possible events that may happen on a shared medium in a randomly chosen slot time [2].
Let $\mathrm{P}_{\text {idle }}, \mathrm{P}_{\text {col }}$ and $\mathrm{P}_{\text {succ }}$ be the probabilities that a randomly chosen slot corresponds to an idle slot, a collision, and a successful transmission, respectively.

Moreover, let $\sigma, \mathrm{T}_{\text {col, }}$ and $\mathrm{T}_{\text {succ }}$ be the duration of the slot corresponding to an idle slot, a collision, and a successful transmission, respectively.

We can obtain the average duration represented by $T_{\text {avg }}$, that a generic slot lasts as follows:

$$
T_{a v g}=P_{i d l e} \delta+P_{\text {succ }} T_{\text {succ }}+P_{c o l}+T_{c o l}
$$

Now, the throughput $\mathrm{S}$ can be calculated as

$$
\begin{gathered}
\begin{array}{c}
\mathrm{S} \\
\text { time }] \ldots . .(2)
\end{array} \quad \text { E[Payload information transmitted in a slot } \\
S=\frac{P_{\text {succ }} x E[P]}{T_{\text {avg }}}=\frac{P_{\text {succ }} x E[P]}{P_{\text {idle }} \sigma+P_{\text {succ }} T_{\text {succ }}+P_{\text {col }} T_{\text {col }}}
\end{gathered}
$$

Where $\mathrm{E}[\mathrm{P}]$ is the average payload size (in terms of time unit), and thus $P_{\text {succ }} x E[P]$ is the average amount of payload information successfully transmitted in a generic slot time.

By dividing the numerator and denominator of equation (2) by $\left(P_{\text {succ }} T_{\text {succ }}\right)$, the throughput can be expressed as follows:

$$
S=\frac{E[P] / T_{\text {succ }}}{1+\frac{P_{\text {col }}}{P_{\text {succ }}} \times \frac{T_{\text {col }}}{T_{\text {succ }}}+\frac{P_{\text {idle }}}{P_{\text {succ }}} \times \frac{\sigma}{T_{\text {succ }}}}
$$

Accordingly, the above analysis applies to both two-way and four way handshakes transmission. To specifically compute the throughput for a given handshake, we only need 
to specify the corresponding values of $\mathrm{T}_{\text {col }}$ and $\mathrm{T}_{\text {succ }}$. Note that the idle slot time, $\sigma$, is specific to the physical layer [3].

\section{NETWORK SIMULATION RESULTS}

A network which has one access point and four nodes was set up as shown below.

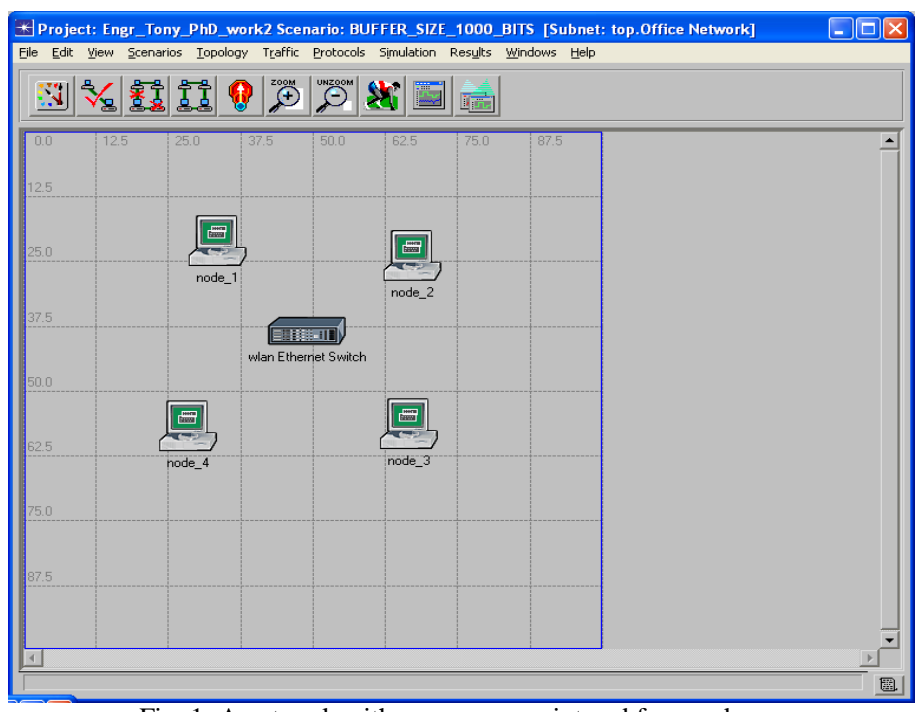

Fig. 1: A network with one access point and four nodes

Simulations were carried out using OPNET IT Guru simulator (Academic edition). The effects of varying three network parameters on the throughput as a performance metric were analyzed. The parameters are: the data-rate, buffer size and fragmentation threshold (FTS).

\section{A. The Data-Rates (Mbps)}

This signifies the speed of the nodes connected within a network. The WLAN model in OPNET IT Guru 9.1 supports data transfer at $1,2,5.5$ and $11 \mathrm{Mbs}$. These data rates are modeled as the speed of transmitters and receivers connected to WLAN MAC process. Each data rate is associated with a separate channel stream, from the MAC process to the transmitter and from the receiver to the MAC process. The values for different data-rates used for the simulation are shown in table 1.

\section{TABLE 1: TABLE SHOWING THE DATA-RATES USED FOR DIFFERENT SCENARIOS}

\begin{tabular}{|l|l|l|l|}
\hline $\begin{array}{l}\text { Attributes } \\
\text { (Parameters) }\end{array}$ & Scenario_1 & Scenario_2 & Scenario_3 \\
\hline Data-rates & 1Mbps & 5.5Mbps & 11Mbps \\
\hline Buffer Sizes & 12800bits & 12800bits & 12800bits \\
\hline $\begin{array}{l}\text { Fragmentation } \\
\text { Threshold }\end{array}$ & None & None & None \\
\hline
\end{tabular}

Based on the simulation of the three scenarios for the datarates, the graphs in figure 1 were obtained. It is found that when the data-rate was increased from $1 \mathrm{Mbps}$ to $11 \mathrm{Mbps}$, the throughput increased. This is predictable from the theoretical view point that as data-rate increases, the number of bits received increases [4].
Thus based on the graphical result below, it can be said that when data-rate increases in a network, the throughput increase; but when the network is overloaded with several stations, that same throughput decreases, since throughput is the number of bits successfully transmitted per second.

The $5.5 \mathrm{Mbps}$ is good for the network, and that is why the graphs first rose sharply before they became stable. Stability of a network is what matters in any network design, and that is why this simulation was performed using long duration of 300 seconds in order to get a good performance study.

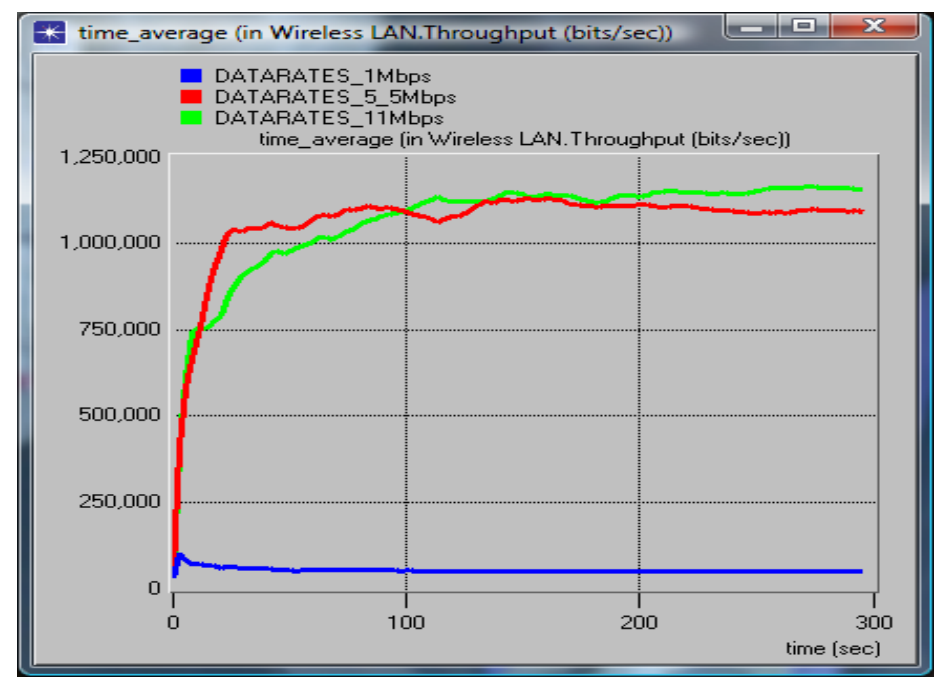

Fig. 2: Throughput study for data-rates of $1 \mathrm{Mbps}, 5.5 \mathrm{Mbps}$ and $11 \mathrm{Mbps}$

\section{B. Buffer Size (bits)}

This parameter specifies the maximum length of the higher layer data arrival buffer. If the buffer limit is reached, data received from the higher layer are discarded until some packets are removed from the buffer so as to have some free spaces to store new packets. The table 2 shows the buffer sizes used.

TABLE 2: TABLE SHOWING THE BUFFER SIZES USED

\begin{tabular}{|l|l|l|l|}
\hline $\begin{array}{l}\text { Attributes } \\
\text { (Parameters) }\end{array}$ & Scenario_1 & Scenario_2 & Scenario_3 \\
\hline Data-rates & $11 \mathrm{Mps}$ & $11 \mathrm{Mbps}$ & $11 \mathrm{Mbps}$ \\
\hline Buffer Sizes & $1000 \mathrm{bits}$ & $6400 \mathrm{bits}$ & $12800 \mathrm{bits}$ \\
\hline $\begin{array}{l}\text { Fragmentation } \\
\text { Threshold }\end{array}$ & None & None & None \\
\hline
\end{tabular}

The graphs of figure 3 show that when the size of the buffer was increased, the throughput increased. For small size of buffer, the throughput reduces to zero, meaning that packets are dropped or discarded because the buffer has no space to accommodate more packets. 


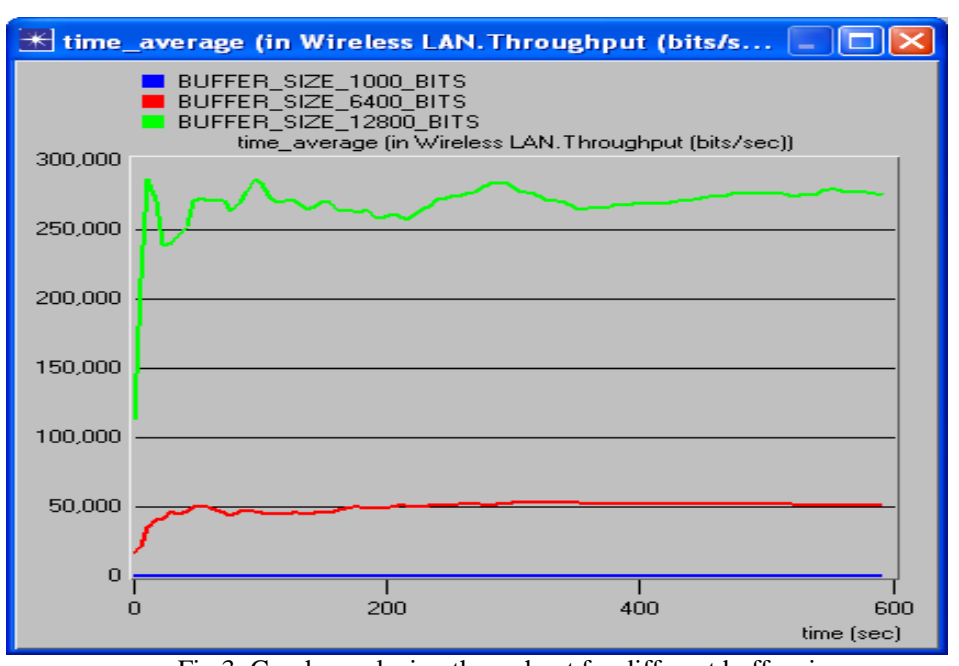

Fig.3: Graphs analyzing throughput for different buffer sizes

\section{Fragmentation Threshold (Bytes)}

This parameter specifies the value to decide if the MAC Service Data Unit (MSDU) received from the higher layers needs to be fragmented before transmission [5]. The number of fragments to be transmitted is calculated based on the size of the MSDU and the fragmentation threshold. Table 3 shows the three scenarios for the simulation study.

The first one is with no fragmentation of incoming packets. The second one is with a fragmentation of 16 bytes, and the third one is with a fragmentation of 256 bytes.

TABLE 3: TABLE SHOWING THE FRAGMENTATION THRESHOLD (FTS) USED FOR DIFFERENT SCENARIOS

\begin{tabular}{|l|l|l|l|}
\hline $\begin{array}{l}\text { Attributes } \\
\text { (Parameters) }\end{array}$ & Scenario-1 & Scenario-2 & Scenario-3 \\
\hline Data-rates & $11 \mathrm{Mps}$ & $11 \mathrm{Mbps}$ & $11 \mathrm{Mbps}$ \\
\hline Buffer Sizes & $12800 \mathrm{bits}$ & $12800 \mathrm{bits}$ & $12800 \mathrm{bits}$ \\
\hline $\begin{array}{l}\text { Fragmentation } \\
\text { Threshold }\end{array}$ & None & 16 bytes & 256 bytes \\
\hline
\end{tabular}

The simulation result in figure 4 indicates that proper fragmentation enhances throughput.

\section{CONCLUSION}

Having completed this simulation, it is seen that when a network parameter is tuned to different scenarios, the throughput performance metric is usually affected. The following points are to be noted from the results of this simulation:

1) When the data-rate in a wireless network is increased, the throughput increases; and packets are delivered more accurately, hence less requirement for retransmission.

2) For a very small size of buffer, if data-rate is increased, the throughput reduces to zero, meaning that packets are dropped or discarded because the buffer has no space to accommodate more packets.

3) Proper fragmentation enhances throughput. But fragmentation increases the size of queue and the number of data dropped in a transmission.

\section{REFERENCES}

[1] Okeshi P.N.," Fundamentals of Wireless Communication", Global Publishers Co., Lagos, Nigeria, 2009.

[2] Achinkole S. O.,"Computer Networks", Orient Printers and Communications, Accra, Ghana, 2010.

[3] Makta M. H., "Basic Computer Communication", Educational Printing \& Publishers, Accra, 2008.

[4] Ede K. I.,"A Guide to Wireless Communication Networks", Excellent Series Printers, Lagos, Nigeria, 2009.

[5] Borah D. K., Daga A., Lovelace G. R., and Deleon P., "Performance Evaluation of the IEEE 802.11a and b WLAN Physical Layer on the Maritain Surface", IEEE Aerospace Conference, Canada, 2005.

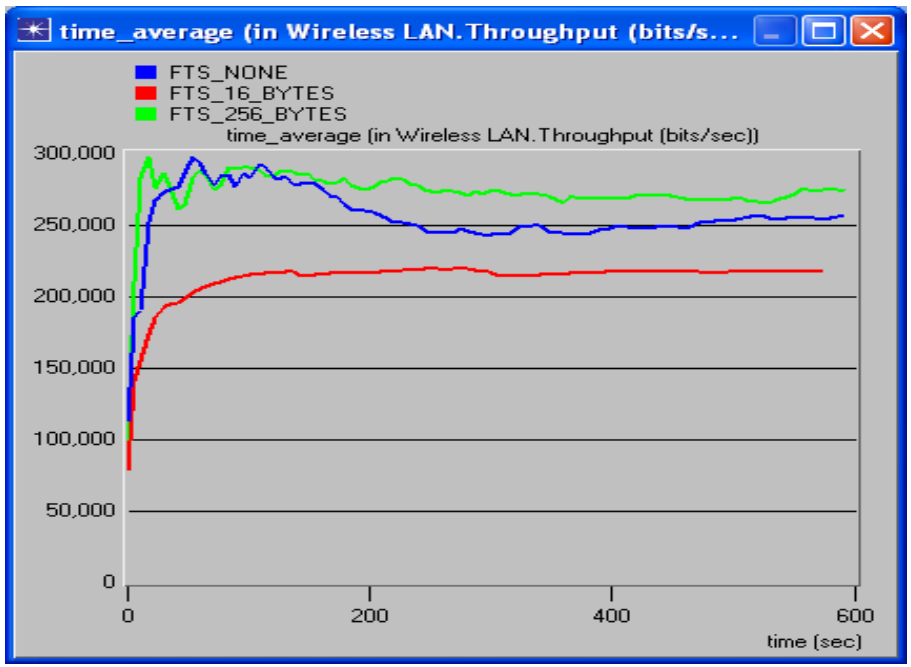

Fig 4: Throughput result for different FTS 